\title{
THE ROTATION CURVES OF GALAXIES
}

\author{
M. S. ROBERTS \\ National Radio Astronomy Observatory*, Green Bank, W. Va., U.S.A.
}

\begin{abstract}
Currently available data on rotation curves are reviewed. For curves derived from optical measurements the distribution of the ratios: the last measured point on a rotation curve to the optical radius of the galaxy has a median value of $\sim \frac{1}{2}$ if Reference Catalogue radii are used and $\sim \frac{1}{3}$ if Holmberg radii are used. It is the absence of easily measurable $H$ II regions that so severely limits the extent of these rotation curves. Accordingly, little can be said of the dependence of $V_{c}$ on $R$ for large $R$, where $R$ is comparable to a Holmberg radius. The assumption that a rotation curve approaches a Keplerian curve after passing its peak rotational velocity implies a strongly concentrated and limited extent of the mass distribution within a galaxy. This assumption is not supported by $21-\mathrm{cm}$ observations of the velocity field within a galaxy. Because of the greater extent of $\mathrm{H}$ I compared to measurable optical (blue) surface brightness, rotation curves may be defined to much larger radii from $21-\mathrm{cm}$ observations. The median value of the above ratio for 14 galaxies is 1.3 . At least 7 of these galaxies show an essentially constant rotational velocity at large $R$, while 5 galaxies have a slowly decreasing $V_{c}(R)$. For both types of curves, a significant surface mass density at large $R$ is required, and a large $(\gtrsim 100)$ mass-to-luminosity ratio is indicated. Such values are consistent with a late dwarf $M$ star population (the most.common type of star in the solar neighborhood) in the outer regions of a galaxy.
\end{abstract}

The first detections of rotation within a galaxy (Slipher, 1914; Wolf, 1914) were made a decade before the definite establishment of the extragalactic nature of many of the nebulae (Hubble, 1924). In the intervening 60 years rotation curves for fewer than 100 galaxies have been measured, a rate of less than two per year. Before the advent of image tubes (and the vast majority of the available data are from this earlier era), the process was difficult and time consuming. An early effort to gather information on the rotation of the inner region of a number of galaxies was made by Mayall (Mayall, 1960; Mayall and Lindblad, 1970). In obtaining spectra of galaxies for redshift measurements he purposefully avoided trailing the image of the galaxy on the spectrograph slit and was able to measure line inclinations for over 50 systems.

But it is the detailed variation of velocity with position over as large an angular extent as possible that is desired. Until the late 1950's such information was available for only a few galaxies. At this time a major observing effort was initiated by the Burbidges. Collaborators and independent observers added to this effort, and much of the information on rotation curves that we now have dates from this era. Another technique for studying the kinematics of galaxies was developing during this same period: observation of the $21-\mathrm{cm}$ spectral line with radio telescopes. This approach has been remarkably improved over this last 15 years through the development of low-noise receivers, the construction of large-filled aperture telescopes, and the application of aperture synthesis techniques.

Examples of all types of galaxies show rotation. For spirals this has long been recognized. Irregular-type galaxies (Ir I) have a chaotic appearance but both optical and $21-\mathrm{cm}$ observations show a well-ordered radial velocity field and well-defined rota-

* Operated by Associated Universities, Inc., under contract with the National Science Foundation. 
tion curves (e.g., NGC 1569, NGC 6822, Ho II, SMC, WLM). The irregulars of type II, such as M82 (Mayall, 1960), also show rotation. There are several examples of rotation within elliptical galaxies, although few such systems have been studied. NGC 4621 (E5) and NGC 4697 (E6) both show inclined spectral lines indicating rotational velocities of 13 and $29 \mathrm{~km} \mathrm{~s}^{-1}(\operatorname{arcsec})^{-1}$, respectively (King and Minkowski, 1965). These measurements cover a diameter of $\sim 10^{\prime \prime}$. Bertola (1972) has extended the measurements for NGC 4697 out to $\sim 40^{\prime \prime}$ (radius) and finds a turnover in the rotation curve near 25". C. Peterson(1975) also finds rotation in a number of elliptical galaxies including the E1 system NGC 3379. Another example, though possibly special, is the high angular velocity of the nucleus of $\mathrm{M} 32,78 \mathrm{~km} \mathrm{~s}^{-1}$ per $10 \mathrm{pc}$ (Walker, 1962).

The bulk of the available data are for spiral galaxies. It is only this category of galaxian rotation curves that are discussed further.

As a measure of the distance to which rotation curves extend, the distribution of the ratio: furthest measured point to optical radius for a number of galaxies is shown in Figure 1. Galaxies in which the observations were restricted to the nuclear region are omitted. The bottom histogram in this figure uses diameters from the Reference Catalogue of Galaxies (de Vaucouleurs and de Vaucouleurs, 1964). A number of these galaxies, shown cross hatched, have diameters measured to a fainter isophote (Holmberg, 1958). These galaxies are replotted in the central histogram using the Holmberg radii to form the ratio. For the first, and smaller, photometric radii the median value of the above ratio is $\sim \frac{1}{2}$. For the Holmberg radii, obviously more realistic since the galaxies extend at least out to this distance, the ratio has a median value of $\sim \frac{1}{3}$. Only one-half the sample of measured rotation curves extend beyond $\frac{1}{3}$ of their photometric radius. And for these the majority are less than $\sim \frac{1}{2}$ the Holmberg radius.

We thus find that the available rotation curves derived from optical studies refer to only a fraction of the photometric extent of a galaxy. The description of the rotation curve beyond the last measured point can only be assumed. Such assumptions may be based on a particular model, such as a constant mass-to-luminosity ratio or an even more extreme case: that the galaxy essentially ends beyond the peak of the rotation curve and the declining branch will quickly approach a Keplerian curve.

Essentially all rotation data are based on emission lines: [O II $], \mathrm{H} \alpha,[\mathrm{N} \mathrm{II}],[\mathrm{S} \mathrm{II}]$, $\mathrm{H}$ I. Absorption lines are generally too faint and subject to possible systematic effects although they are used in the measurement of line inclinations for rotation periods of the central section of a galaxy. The $21-\mathrm{cm}$ data are especially valuable for measurable $\mathrm{H}$ I radiation is found at a much larger radial distance than optical emission lines. Thus far, only radio measurements are able to supply information on the rotation of the outer part of a galaxy.

In the sample of optically-studied galaxies M31 (Rubin and Ford, 1970) extends the furthest in linear extent, $24 \mathrm{kpc}$, a value 1.2 the Holmberg radius. Their derived rotation curve shows no indication of a decline in the circular velocity over the outermost $4 \mathrm{kpc}$ (Rubin and Ford, 1970, especially page 389). A similar finding of constant circular velocity, but now out to $30 \mathrm{kpc}$, appears in $21-\mathrm{cm}$ observations of M31 (Roberts and Rots, 1973; Roberts and Whitehurst, 1975). The ratio of this distance 
to the Holmberg diameter is 1.5 . The greater extent of the neutral hydrogen compared to Holmberg's limiting blue isophote ( $26^{m} .5$ per sq arcsec) has long been recognized and is clearly shown by the top histogram in Figure 1 where 11 of 14 galaxies have a ratio greater than one. As noted earlier, it is this more extensive $\mathrm{H}_{\mathrm{I}}$ distribution that allows rotation curves to be derived to angular distances compa-

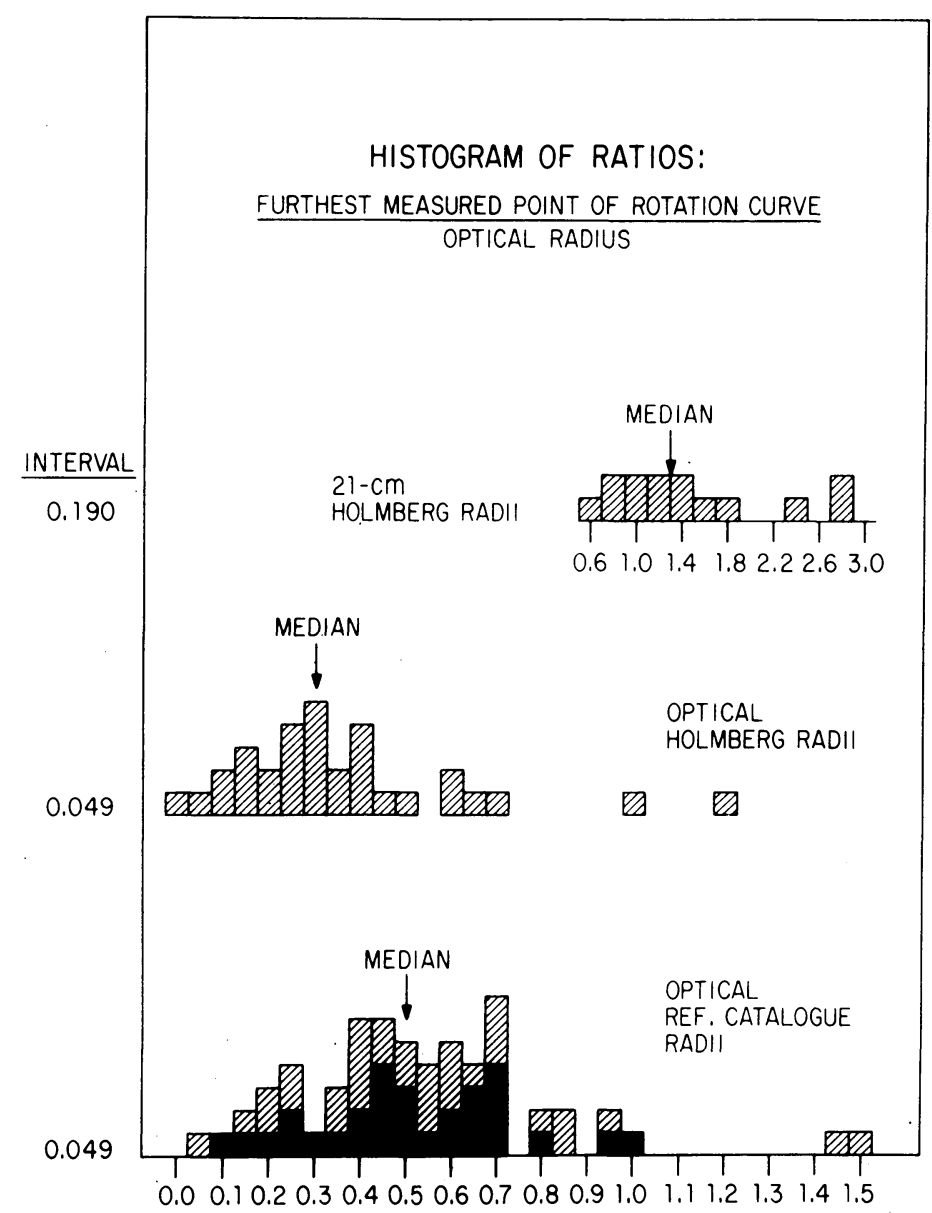

Fig. 1. Distribution of the extent of rotation curves in terms of optical radii. The bottom histogram is based on optical determinations of rotation curves and radii taken from the Reference Catalogue of Bright Galaxies. Those galaxies which also have radii measured by Holmberg are indicated by hatched lines; they are represented in the center histogram using the Holmberg radii to form the ratio. The top histogram is based on rotation curves derived from $21-\mathrm{cm}$ measurements and Holmberg radii.

rable to or greater than the Holmberg radius. A limiting factor in such work is the relative resolution: beam size/H I size, available in $21-\mathrm{cm}$ observations. Filled aperture, circular half-power beam widths of 9' (100-m telescope) and 10' (300-ft telescope) are now available. The recently resurfaced Arecibo antenna will have a $4^{\prime}$ beam. The 


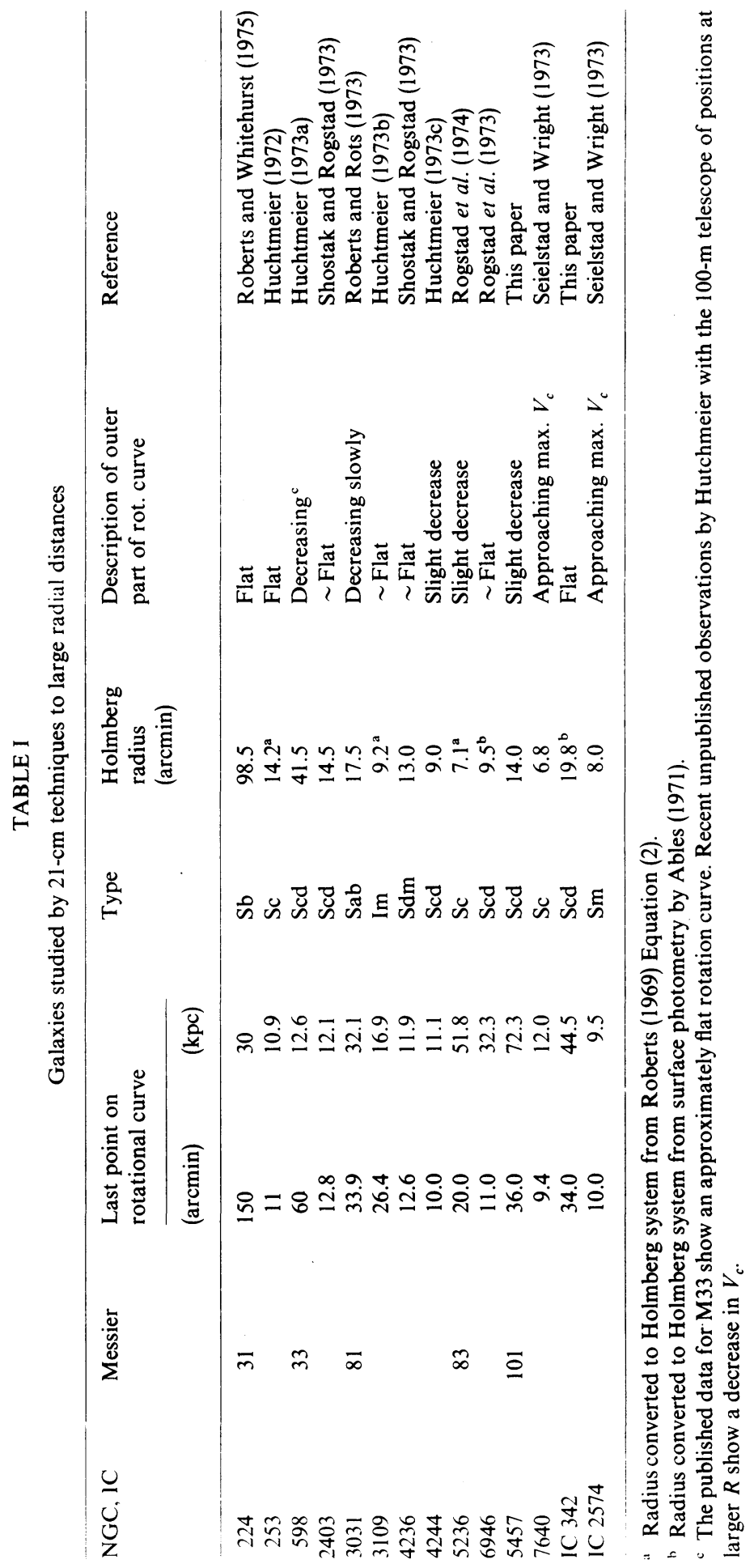


Nancay telescope has a fan-beam of $4^{\prime} \times \sim 25^{\prime}$ (the latter dimension is dependent on the declination of the source). These instruments are particularly sensitive to low surface brightness radiation, i.e., low $\mathrm{H}_{\mathrm{I}}$ column density, and are therefore particularly suited for such measurements. Their relatively large beams limit the number of galaxies that can be studied for this purpose. Successful observations with synthesized beams as small as $25^{\prime \prime}$ have been made at Westerbork. The Cal Tech interferometer has been used to study a number of galaxies with an effective resolution of $2^{\prime}$ and the Cambridge $\frac{1}{2}$-mile interferometer with comparable resolution. Unless an inordinate amount of observing time is used, these instruments are seriously limited in the surface brightness that is measurable. Instrumental effects may also be greater: in addition to the side lobes of the main beam (common to all telescopes) there are side lobes of the synthesized beam as well as a grating response. However, the relatively high angular resolution that is attainable is ideal for studies of the kinematics and hydrogen distribution in the inner region of a galaxy, i.e., out to $\sim 1$ Holmberg radius.

Twenty-one centimeter data based on good relative resolution are available for 14 galaxies listed in Table I. We exclude the Magellanic Clouds where the data are of high quality and extensive but in the present context ambiguous (Hindman, 1967; McGee and Milton, 1966), and IC10 (Shostak, 1974) where only a small velocity gradient is observed. Several of the 14 systems have been studied by more than one group, only the most complete or extensive references are included.

Examples of some of these rotation curves are shown in Figures 2 (M31), 3 (M101), and 4 (IC 342). The first is taken from Roberts and Whitehurst (1975) and shows for $R>12 \mathrm{kpc}$ the optically measured data points (Rubin and Ford, 1970) and the 21-cm data points. For M101 and IC 342 recently derived data obtained with the $300-\mathrm{ft}$

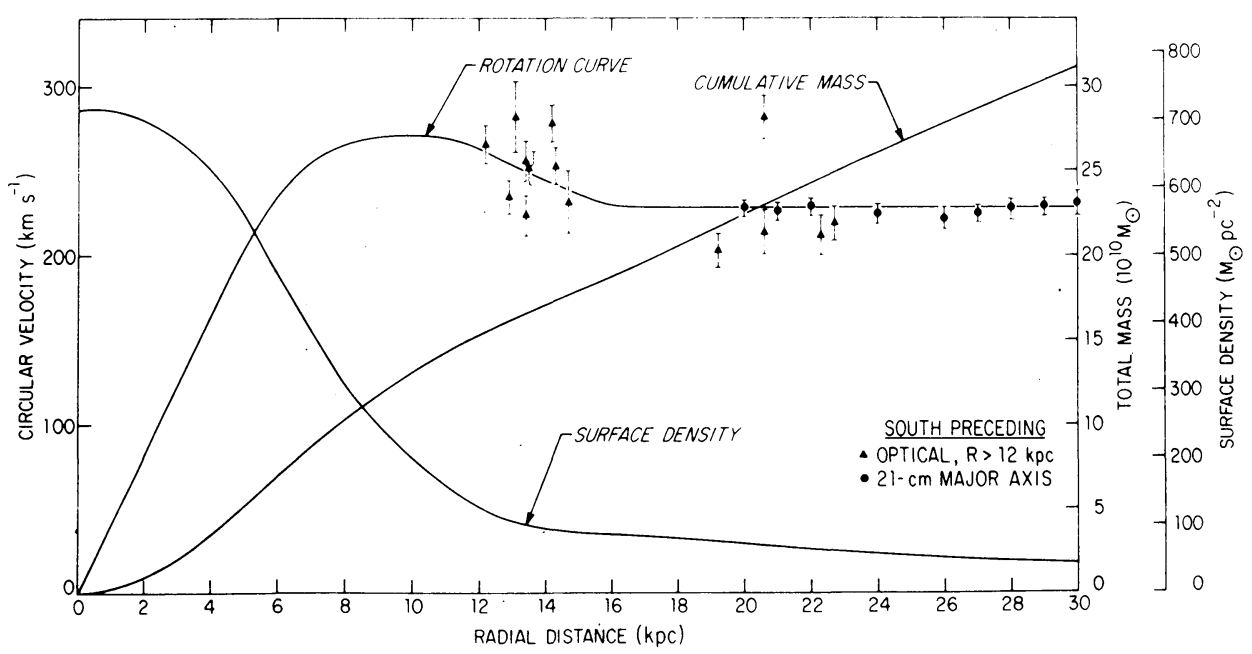

Fig. 2. The rotation curve for M31. For simplicity the inner peak of the rotation curve near $0.8 \mathrm{kpc}$ is omitted. The filled triangles are optically determined rotational velocities from Rubin and Ford (1970) for the outer $12 \mathrm{kpc}$. The filled circles are $21-\mathrm{cm}$ measurements made with the $300-\mathrm{ft}$ telescope. The surface density and cumulative mass curves are for a thin disk model. 


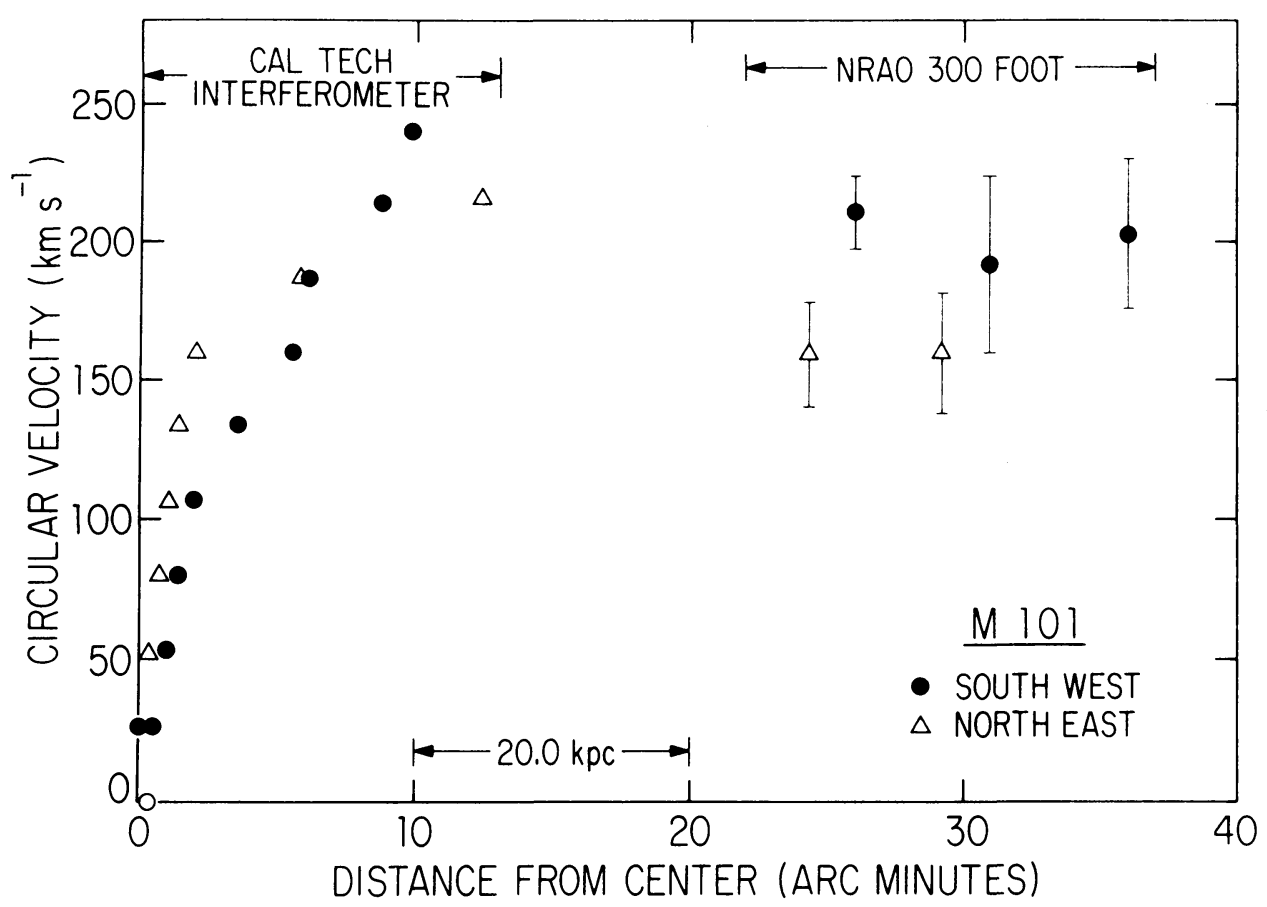

Fig. 3. The rotation curve for M101. All data points are based on $21-\mathrm{cm}$ observations. The inner $12^{\prime}$ are from interferometer measurements by Rogstad and Shostak (1971) and are for major axis values in their Figure 4. The outer points are from recent measurements made with the $300-\mathrm{ft}$ telescope.

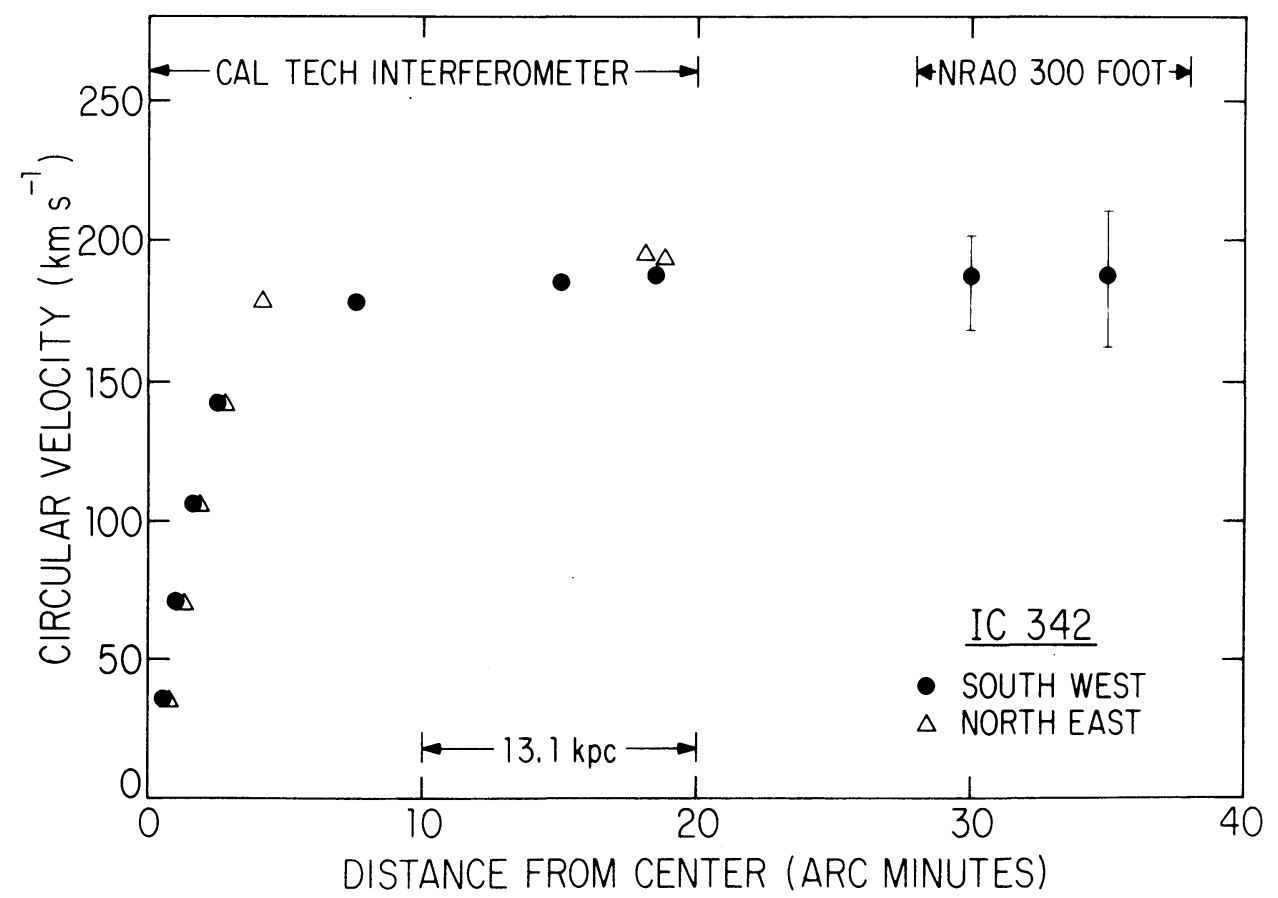

Fig. 4. The rotation curve for IC 342. All data points are based on $21-\mathrm{cm}$ observations. The inner 19' are from interferometer measurements by Rogstad et al. (1973) and are for major axis values in their

Figure 5. The outer points are from recent measurements made with the $300-\mathrm{ft}$ telescope. 
telescope are added to inner portions of rotation curves obtained with the Cal Tech interferometer (Rogstad and Shostak, 1971; Rogstad et al., 1973). At least 2 of the 3 curves show a relatively constant circular velocity at large $R$. Of the 14 galaxies, 7 show an essentially constant rotational velocity at large radius, near or beyond the Holmberg radius. In some instances there is even a suggestion that after the flat portion of the rotation curve there may be an increase in $V_{c}$ with $R$; M31 is one such example. Of the remaining seven rotation curves, two have not yet reached a turnover at the maximum velocity, NGC 7640 and IC 2574. The other five rotation curves show, in varying amounts, a gradual decrease. This is most pronounced in M81, an Sab system and the earliest type galaxy in Table I. It is important to note that this sample is heavily weighted to late-type systems. Only 2 of the 14 are earlier than Sc; these are M31 (Sb) and M81 (Sab).

For any specific case, there are a number of possible observational effects that can be invoked to account for the flat rotation curves. The most obvious relates to side lobes sensing radiation from a region away from the direction of the primary beam. However, for the 300-ft observations of M31, the side lobes are carefully measured to $-30 \mathrm{~dB}$. At a position 140 arc min along the south-preceding major axis, such side lobe contributions are $\sim 3 \%$ of the observed signal. The velocity profile at this position obtained with the 140-ft telescope, the Jodrell Bank 250-ft telescope (Gottesman and de Jager, 1970), as well as a profile constructed from maps obtained with the Nancay antenna (Guibert, 1970) all yield velocities in substantial agreement with that obtained with the 300 -ft telescope. We further note that among the various galaxies with flat rotation curves, some were derived with the $\mathrm{Cal}$ Tech interferometer, some with the Nançay tiltable plane-standing parabola, and some with the NRAO 300-ft antenna. And equally important for possible instrumental effects the sample of galaxies obviously covers a wide range of position angles and angular sizes. Nevertheless, the Cambridge group (Emerson and Baldwin, 1973) derive a rotation curve for M31 which is slowly decreasing and thus differs from the rotation curve derived from 300-ft data. It is difficult to reconcile their rotation curve with the one in Figure 2. The outer and most critical points defining their curve fall at the edge and even outside the velocity range in which a signal is measured with the 300 -ft telescope. Angular resolution effects due to the different size beams will not account for these differences. The data at the representative point of 140 arcmin along the south-preceding major axis obtained with 4 different filled aperture telescopes are consistent. We conclude that Figure 2 is indeed descriptive of the motion in the outer part of M31.

(Observations of M31 made with the 100-m telescope after this paper was presented at Besançon are in complete agreement with the 300-ft data. The Emerson and Baldwin (1973) rotation curve appears to be based on spurious data even though the 'error' bars on the points defining their rotation curve are remarkably small.)

The 14 rotation curves in Table I are derived from radiation arising from the general interstellar medium. The optical data refer (primarily) to discrete $\mathrm{H}$ il regions. Could these two subsystems of a galaxy have different kinematic properties. On a 
small scale (parsecs) this is possible; on the dimensional scale considered here it is very unlikely. Support for this latter conclusion comes from detailed comparison of $\mathrm{H}$ I and $\mathrm{H}$ II velocity measurements, e.g., SMC, LMC, M31 and M81. Both neutral and ionized hydrogen may be considered as equivalent test 'particles' of the kinematic field within a galaxy.

The dependence of total mass with radius $R, M(R)$, varies as $\sim R$ for those cases and regions where the rotation curve has a constant circular velocity. But at least $\frac{1}{3}$ of the galaxies studied thus far have $V_{c}$ decreasing with $R$, albeit very gradually. In all cases there is a significant contribution to the total mass from large $R$, a component whose optical surface brightness is very small, indicating a large mass-to-light ratio in the outer parts of the galaxies studied here. Although $\mathrm{H}$ I extends well beyond the Holmberg radius for these galaxies, its surface density is only $\sim 1 \%$ of the total mass surface density implied by the rotation curves. The most common type of star in the solar neighborhood, intermediate and late dwarf $M$ stars could easily satisfy both the mass surface density requirement and the upper limits of the optical surface brightness. As an example, in M31 at $28 \mathrm{kpc}$ from the center, the surface density (as computed for a thin disk model) is $\sim 50 M_{\odot} \mathrm{pc}^{-2}$. This location is beyond the limit of de Vaucouleurs's (1958) photometry of M31 but an extrapolation of his data indicate $M / L_{p g} \simeq 400$. The implication of a change in the luminosity function with radial distance from the center of a galaxy need not be alarming. We know this to be the case by the very appearance of spiral arms (see also McCuskey, 1965). In the solar vicinity the shape of the luminosity function changes significantly with height above the galactic plane (Bok and MacRae, 1941). This change is such that at high $z$ $(1 \mathrm{kpc})$ there are relatively more late type stars per volume of space. This is the sense of the change required to explain the shape of the rotation curves at large $R$.

The picture that emerges from these data is one in which spiral galaxies are significantly more extensive than indicated by blue limiting exposures. The projected surface density in these outer regions decreases approximately linearly with distance. The necessary mass and the implied mass-to-luminosity ratio can be attributed to those stars found near the peak of the local luminosity function, late-type dwarfs. This is not a unique explanation; it is the simplest and is susceptible to observational confirmation through near infrared observations.

\section{References}

Ables, H. D.: 1971, Pub. U.S. Naval Obs. 2nd Series, 2, No. 4, Washington, D.C.

Bertola, F.: 1972, Osservatorio Astronomico di Padova Comunicazioni E Rassegne, No. 98.

Bok, B. J. and MacRae, D. A.: 1941, in E. M. Schlaikjer (ed.), The Fundamental Properties of the Galactic System, Annals of the New York Academy of Sciences 42, 219.

Emerson, D. T. and Baldwin, J. E.: Monthly Notices Roy. Astron. Soc. 165, 9P.

Gottesman, S. T. and de Jager, G.: 1970, Mem. Roy. Astron. Soc. 74, 67.

Guibert, J.: 1973, Astron. Astrophys. Suppl. Series 12, 263.

Hindman, J. V.: 1967, Australian J. Phys. $20,147$.

Holmberg, E. : 1958, Medd. Lund, Ser. II, No. 136.

Hubble, E.: 1924, Paper read at the 33rd Meeting of the American Astronomical Society. Abstracted in Popular Astron. 33, 252, 1925. 
Huchtmeier, W.: 1972, Astron. Astrophys. 17, 207.

Huchtmeier, W.: 1973a, Astron. Astrophys. 22, 91.

Huchtmeier, W.: 1973b, Astron. Astrophys. 22, 27.

Huchtmeier, W.: 1973c, Astron. Astrophys. 23, 93.

King, I. R. and Minkowski, R.: 1966, Astrophys. J. 143, 1002.

McCuskey, S. W.: 1965, Stars and Stellar Systems 5, 1.

McGee, R. X. and Milton, J. A.: 1966, Australian J. Phys. 19, 343.

Mayall, N. U.: 1960, Ann. Astrophys. 23, 344.

Mayall, N. U. and Lindblad, P. O.: 1970, Astron. Astrophys. 8, 364.

Peterson, C.: 1975, 'Stellar Motions in Elliptical Galaxies', Univ. of Calif., Berkeley (Ph.D. Thesis).

Roberts, M. S. : 1969, Astron. J. 74, 859.

Roberts, M. S. and Rots, A. H.: 1973, Astron. Astrophys. 26, 483.

Roberts, M. S. and Whitehurst, R. N.: 1975, Astrophys. J. (submitted).

Rogstad, D. H. and Shostak, G. S.: 1971, Astron. Astrophys. 13, 99.

Rogstad, D. H., Lockhart, I. O., and Wright, M. C. H.: 1974, Astrophys. J. (in press).

Rogstad, D. H., Shostak, G. S., and Rots, A. H.: 1973, Astron. Astrophys. 22, 111.

Rubin, V. C. and Ford, K. W., Jr.: 1970, Astrophys. J. 159, 379.

Seielstad, G. A. and Wright, M. C. H.: 1973, Astrophys. J. 184, 343.

Shostak, G. S.: 1974, Astron. Astrophys. 31, 97.

Shostak, G. S. and Rogstad, D. H.: 1973, Astron. Astrophys. 24, 405.

-Slipher, V. M.: 1914, Lowell Obs. Bull. No. 62.

Vaucouleurs, G. de: 1958, Astrophys. J. 128, 379.

Vaucouleurs, G. de and Vaucouleurs, A. de: 1964, Reference Catalogue of Bright Galaxies, Univ. of Texas Press, Austin.

Walker, M. F.: 1962, Astrophys. J. 136, 695.

Wolf, M.: 1914, Vierteljahrsschieft der Astr. Gesellsch. 49, 162.

\section{DISCUSSION}

Freeman: Did you say a surface density of $50 M_{\odot} \mathrm{pc}^{-2}$ ?

Roberts: Yes.

Freeman: This. means an $M / L$ way over 100 to go below de Vaucouleurs photometry.

Roberts: The mass-to-light ratio is indeed high, several hundred. Note that I use photographic luminosity in these considerations.

Bertola: Did you find any relation between the shape of the rotation curve and the behaviour of $M / L$ within the galaxy?

Roberts: There is a correlation of the general form of a rotation curve with type, earlier galaxies are more centrally concentrated. But there are too few curves as yet, to look for a shape vs $M / L$ relation.

Baldwin: What are the Holmberg radii in NGC 2403 and 4236 relative to the limits of the $\mathrm{H}$ I observations at about $12^{\prime}$ radius.

Roberts: For both galaxies the observed rotation curves extend to just less than a Holmberg radius.

Gott: As far as extrapolations of the rotation curves are concerned. The total mass of M31 may be estimated by an examination of the dynamics of our galaxy and M31, namely that they have just turned around and are approaching each other due to their mutual gravitational attraction. This fact together with the distance to M31 gives a total mass for the Milky Way plus M31 system. This indicates a $(M / L)_{B} \sim 100$ consistent with your values and would allow a continuation of a flat rotation curve to approximately twice the distance shown by the radio data so far.

King: Larger masses for galaxies would help to solve the other great 'missing mass' problem, that in clusters of galaxies. Evidence is accumulating that in the Coma cluster, where about a factor of 8 is needed, the missing mass is actually in the galaxies.

Lynden-Bell: Smart, a student at Cambridge looking at 11 So satellites of one of the giants in Coma points out that they form an isolated system of So's and gives a mass of $2 \times 10^{13} M_{\odot}$ for the giant. The So's are essentially embedded in the giant so there is no doubt that at least this mass is in the giant galaxy.

Gott: I might mention two additional pieces of information which also support the viewpoint that the mass resides primarily in the galaxies. Some time ago Dr Gunn and I estimated the mass of the two largest galaxies in the Coma cluster. These galaxies dominate the center of the cluster and therefore can be treated 
as a binary system and their masses estimated from their separation and relative radial velocities. These estimates also give several times $10^{13} M_{\odot}$ for the masses of these galaxies in agreement with your figure. A second point is that Gus Omeler has recently done a study of the degree of central condensation of galaxies in the Coma cluster and found that for the brighter galaxies there was greater central concentration, but for the fainter galaxies there was no effect. Comparison with models by Aarseth showed that this was just the effect expected from two-body relaxation if the brightest galaxies had mass to light ratios equal to the total mass to light ratio for the cluster.

Lecar: Theoretical models by Gott, Larson of formation of galaxies have difficulty obtaining space density fall offs as shallow as $r^{-2.5}$ to $r^{-3}$. At this time, no model has produced $\varrho \sim r{ }^{2}$ (isothermal sphere) or $M \sim r$ as indicated by the flat rotation curves Roberts has just shown us. 